\title{
El ácido valproico como agente sensibilizador al tratamiento
} anticáncer

Gabriela Rebeca Luna-Palencia, ${ }^{1}$ José Correa-Basurto² y Ismael Vásquez-Moctezuma ${ }^{3 *}$

${ }^{1}$ Centro de Investigación y de Estudios Avanzados-Instituto Politécnico Nacional, Departamento de Biotecnología; ${ }^{2}$ Instituto Politécnico Nacional, Escuela Superior de Medicina, Sección de Estudios de Posgrado e Investigación, Laboratorio de Modelado Molecular y Diseño de Fármacos; ${ }^{3}$ Instituto Politécnico Nacional, Escuela Superior de Medicina, Sección de Estudios de Posgrado e Investigación. Ciudad de México, México

\section{Resumen}

El ácido valproico es un fármaco antiepiléptico con más de 50 años de uso clínico. En la década pasada se descubrieron sus efectos anticancerígenos. El análisis de grupos de pacientes que utilizaron este fármaco durante años ha mostrado que disminuye la frecuencia de cáncer de cabeza y cuello. Estudios recientes evidencian el efecto anticáncer al combinar el ácido valproico con la quimioterapia, terapia biológica e inhibidores de sistemas antioxidantes, con resultados excepcionales. En esta revisión se analiza el metabolismo del ácido valproico y su aplicación contra el cáncer.

PALABRAS CLAVE: Acido valproico. Terapia del cáncer humano. Terapia epigenética. Inhibidores de desacetilasas de histonas.

\section{Abstract}

Valproic acid is an antiepileptic drug with more than 50 years of clinical use. In the past decade, its anticancer effects were discovered. Analyses in groups of patients who used this drug for years have shown that it decreases the frequency of head and neck cancer. Recent studies show the anticancer effect of combining valproic acid with chemotherapy, biological therapy and antioxidant systems inhibitors, with exceptional results. In this review, we analyze the metabolism of valproic acid and its application against cancer.

KEY WORDS: Valproic acid. Human cancer therapy. Epigenetic therapy. Histone deacetylase inhibitors.

\section{Introducción}

El ácido valproico (AVP) se utiliza en enfermedades neurológicas como epilepsia, migraña, trastorno bipolar y déficit de atención. ${ }^{1,2}$ El AVP funciona como sensibilizador a los tratamientos anticáncer al modificar epigenéticamente la expresión de genes.

\section{Epigenética y cáncer}

Las neoplasias malignas pierden la capacidad para controlar su proliferación, invadiendo otros tejidos (metástasis). ${ }^{3}$ Esta alteración se debe a mutaciones en los protooncogenes y genes supresores de tumores. Lo anterior es generado por cambios genéticos
Gac Med Mex. 2019;155:417-422

Disponible en PubMed www.gacetamedicademexico.com 
y epigéneticos. ${ }^{3,4}$ Los cambios epigenéticos pueden ser reversibles y consisten en mecanismos como la metilación de las regiones ricas en citocinas (CpG) del ADN o "islas CpG" que al ser metiladas inhiben la expresión de genes, el bloqueo del ARN mensajero por moléculas de micro-ARN (miARN) y la desacetilación de histonas. ${ }^{3,4}$

\section{Desacetilasas y acetiltransferasas de histonas en el control epigenético de los genes}

El ADN se enrolla en los octámeros de histonas $(\mathrm{H} 2 \mathrm{~A}, \mathrm{H} 2 \mathrm{~B}, \mathrm{H} 3 \mathrm{y} \mathrm{H} 4)$ que forman el nucleosoma, que cuando se condensan integran la cromatina. ${ }^{5}$ Cada histona tiene una "cola" en el amino terminal rica en aminoácidos básicos como la lisina, blanco de modificaciones postraduccionales, de tal forma que la accesibilidad al ADN es en parte controlada por cambios en esta estructura. ${ }^{6}$ Los mecanismos de regulación de histonas incluyen la modificación por metilación, acetilación, fosforilación y ubiquitinación, entre otros. ${ }^{7} \mathrm{La}$ acetilación y desacetilación de histonas y proteínas citoplásmicas son reversibles y se controlan mediante dos enzimas: las acetiltransferasas de histonas y las desacetilasas de histonas (HDAC). ${ }^{8}$ Las acetiltransferasas de histonas transfieren acetilos a las lisinas de las colas de histonas, lo que elimina la carga positiva de las lisinas, disminuyendo la unión con el ADN. Con lo anterior, acceden factores de transcripción y la ARN polimerasa. ${ }^{7,8}$ Por el contrario, las HDAC remueven grupos acetilo, aumentando la atracción del ADN hacia las cargas positivas de las histonas; este ADN compactado no permite la entrada de factores de transcripción ni de la ARN polimerasa. En el cáncer se desacetilan preferentemente los genes supresores. ${ }^{7,8}$ La acetilación también controla las proteínas citoplásmicas, regulando la expresión de genes, ciclo celular, corte y empalme (splicing), transporte y nucleación de actina. ${ }^{9}$

\section{La familia de las HDAC}

En el humano se conocen 11 HDAC, divididas en las clases I, II, III y IV, cuya clasificación se basa en su homología con las HDAC de levadura: ${ }^{6}$

- HDAC clase I, proteínas que usan $\mathrm{Zn}^{+}$como cofactor y se expresan de manera ubicua e incluyen a HDAC1, HDAC2, HDAC3 y HDAC8.

- HDAC clase lla incluyen a HDAC4, HDAC5, HDAC7 y HDAC9; se encuentran tanto en el núcleo como en el citoplasma,
- HDAC clase llb están presentes en el citoplasma e incluyen a HDAC6 y HDAC10.

- HDAC clase III, sirtuinas, se localizan en el citoplasma y mitocondria; ocupan el dinucleótido de nicotinamida y adenina como cofactor.

- HDAC clase IV incluyen a HDAC11 y se ubican en el citoplasma ${ }^{10,11}$ (Tabla 1).

HDAC1, HDAC2, HDAC3 y HDAC8 participan en la proliferación celular. En la apoptosis destacan HDAC1 y HDAC2. En la resistencia a la quimioterapia predomina HDAC1; en la diferenciación, HDAC3, HDAC4, HDAC5 y HDAC8; en la angiogénesis, HDAC4, HDAC6, HDAC7 y HDAC10; y en la migración, HDAC6. ${ }^{12,13}$

\section{Metabolismo del ácido valproico}

El AVP en niños se ha asociado con insuficiencia hepática. ${ }^{14-16}$ Su eliminación depende de su biotransformación en productos más solubles en agua, proceso que se divide en dos fases: ${ }^{14}$

- Fase I, incluye reacciones de oxidación, reducciones e hidrólisis.

- Fase II, participan reacciones de conjugación con glucuronato, glutatión, carnitina, coenzima A o aminoácidos como glicina o ácido glutámico.

El metabolismo oxidativo del AVP es mitocondrial por medio de la betaoxidación. En el hepatocito se inactiva por medio de la fase II, que por conjugación genera productos polares para su excreción renal. El AVP es un sustrato para las isoformas CYP2C6 y CYP2C9. ${ }^{17,18} \mathrm{De}$ todos los compuestos del metabolismo que se generan, el 4-ene-valproico es más hepatotóxico (Figura 1). ${ }^{16}$

\section{Mecanismo de acción de los inhibidores de HDAC}

Los inhibidores de HDAC (iHDAC), como el AVP, detienen el ciclo celular, generan diferenciación y apoptosis en las líneas celulares cancerígenas en el humano, inhiben el crecimiento tumoral en modelos animales y tienen actividad antitumoral en ensayos clínicos controlados. ${ }^{19-21}$ Además, activan la autofagia, generan especies reactivas de oxígeno e interrumpen la vía del agresoma. ${ }^{13}$ La inhibición de las HDAC provoca sobreacetilación de estas proteínas, lo que reactiva la transcripción de genes supresores de tumores y revierte el cáncer. ${ }^{6}$

En estudios clínicos controlados, los iHDAC muestran resultados aceptables en el tratamiento de neoplasias hematológicas, por lo que en 2006 la Food and Drug Administration aprobó el ácido hidroxámico 
Tabla 1. Clasificación de las desacetilasas de histona

\begin{tabular}{|c|c|c|}
\hline Grupo de HDAC & Enzima HDAC & Proteínas con las que interacciona \\
\hline \multirow[t]{4}{*}{$\begin{array}{l}\text { Clase I } \\
\text { (homóloga a Rpd3) }\end{array}$} & HDAC1, HDAC2, HDAC3, HDAC8 & $\begin{array}{l}\text { p53, RB, MYOD, NF-kB, DNMT1, DNMT3a, MBD2, Sp1, BRCA1, MeCP2, } \\
\text { ATM, Smad7 }\end{array}$ \\
\hline & HDAC2 & $\mathrm{RB}, \mathrm{NF}-\kappa \mathrm{B}, \mathrm{BRCA} 1, \mathrm{DNMT1}$ \\
\hline & HDAC3 & RB, NF-кB , Smad7, Stat3, SRY \\
\hline & HDAC8 & ND \\
\hline \multirow{4}{*}{$\begin{array}{l}\text { Clase lla } \\
\text { (homólogo a Hdac) }\end{array}$} & HDAC4 & MEF2 \\
\hline & HDAC5 & MEF2 \\
\hline & HDAC7 & MEF2 \\
\hline & HDAC9 & \\
\hline \multirow[t]{2}{*}{ Clase IIb } & HDAC6 & Smad7, $\alpha$-tubulina, Hsp-90 \\
\hline & HDAC10 & \\
\hline \multirow{7}{*}{$\begin{array}{l}\text { Clase III } \\
\text { (homóloga a Sir2) }\end{array}$} & SIRT1 & p53, FOX01, p300, NF-kB, $\alpha$-tubulina \\
\hline & SIRT2 & $\alpha$-tubulina \\
\hline & SIRT3 & \\
\hline & SIRT4 & \\
\hline & SIRT5 & \\
\hline & SIRT6 & Relacionada con la heterocromatina \\
\hline & SIRT7 & Relacionada con el nucleolo \\
\hline $\begin{array}{l}\text { Clase IV } \\
\text { (similar a clases I y II) }\end{array}$ & HDAC11 & \\
\hline
\end{tabular}

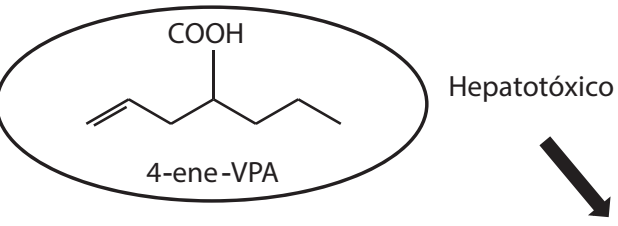<smiles>CCCC(CCC)C(=O)O</smiles>

Metabolismo del citocromo P 450

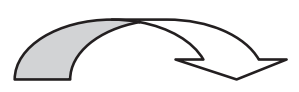

CYP2C9

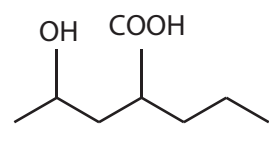

Esteatosis microvesicular

4-OH-VPA<smiles>CCCC(CCCO)C(=O)O</smiles>

5-OH-VPA

Figura 1. Biotransformación del ácido valproico por el sistema de citocromos; se muestra la formación del metabolito hepatotóxico 4-ene-valproico.

suberoilanilida (vorinostat y SAHA) y en 2009 la romidepsina para el tratamiento del linfoma cutáneo de células $\mathrm{T}$, la displasia de médula ósea y el linfoma periférico de células T. ${ }^{22} \mathrm{~A}$ inicios de 2015 fue aprobado el panobinostat para el manejo del mieloma múltiple. ${ }^{23}$ No obstante lo anterior, en tumores sólidos los 
resultados han sido variables. ${ }^{24}$ Los iHDAC también pudieran ser útiles en enfermedades virales y diabetes mellitus 2. ${ }^{23-27}$ Se han observado buenos resultados en linfoma folicular y linfoma de zona marginal. ${ }^{28}$

Originalmente se observó que el AVP inhibe la proliferación celular del neuroblastoma murino y del glioma; también se ha encontrado que la exposición continua a AVP induce diferenciación de líneas celulares y apoptosis. ${ }^{29-32}$ El AVP se ha clasificado como un inhibidor selectivo de las HDAC clase I. ${ }^{24,33}$

\section{Modelado in silico del AVP sobre la HDAC8}

El mecanismo por el cual el AVP inhibe a las HDAC no se conoce, sin embargo, un estudio in silico con HDAC8 utilizando el AVP como ligando sugiere que hay dos sitios de unión: el sitio catalítico y el canal hidrofóbico del sitio activo. El grupo carboxilo del AVP interactúa con el sitio catalítico. Por otro lado, se piensa en el canal hidrofóbico del sitio activo se libera el acetato bloqueando a la enzima. ${ }^{34}$

\section{El AVP sensibiliza al cáncer y coadyuva con la quimioterapia}

El AVP en la terapia del cáncer se ha aplicado como monoterapia o en combinación con agentes epigenéticos desmetilantes, quimioterapia y moduladores del sistema inmunitario. ${ }^{35}$ En una investigación de fase II se observó que como monoterapia en carcinoma neuroendocrino era capaz de inducir la expresión de Notch I (supresor de tumores); el estudio incluyó a ocho pacientes, uno respondió parcialmente y cinco evolucionaron a enfermedad estable. ${ }^{36}$ In vitro, en neoplasias mieloides ha mostrado inducción de apoptosis y diferenciación en las células leucémicas no diferenciadas, lo que ha estimulado el uso del AVP como monoterapia o en combinación con el ácido transretinoico total en leucemia mieloide aguda (AML) y el síndrome mielodisplásico (MDS). En un estudio de fase II con 75 pacientes tratados con AVP asociado con el ácido transretinoico total se reportó que 18 pacientes (24\%) lograron respuestas adecuadas. En otro estudio con AVP y ácido transretinoico total se trataron 20 pacientes con MDS: se observó beneficio clínico en $30 \%$ de los pacientes con AML y MDS. ${ }^{37,38}$ En otro enfoque se utilizó 5-azacitidina más AVP, reportando mejor respuesta que la terapia convencional en estudios fases I, II y III en adultos mayores con AML y MDS. ${ }^{37,39,40}$ En un estudio de fase II de AVP con ácido transretinoico total y 5-azacitidina aplicados a pacientes con AML y con MDS de riesgo bajo se observó respuestas en $23 \%$ de los pacientes y una supervivencia de 12.4 meses. ${ }^{41}$

Las combinaciones del AVP con quimioterapia incluyen agentes que dañan al ADN. En un estudio en el que se combinó con epirubicina (inhibidor de la topoisomerasa II) se observó respuestas en $22 \%$ de los 44 pacientes incluidos, entre ellos pacientes con tumores considerados resistentes a las antraciclinas, como los melanomas. ${ }^{42}$ En un estudio fase I-II se combinó AVP con 5-fluorouracilo, epirubicina y ciclofosfamida en una cohorte de 15 pacientes con cáncer de mama; en $64 \%$ de los pacientes se observó toxicidad aceptable. ${ }^{35}$ En un estudio clínico fase II de 16 pacientes con mesotelioma maligno inoperable y resistente al cisplatino se detectaron resultados sinérgicos con la combinación de AVP y doxorubicina; siete pacientes de 45 mostraron respuesta parcial. ${ }^{43}$ En otro trabajo clínico de fase I/II de pacientes con melanoma metastásico se utilizó AVP en combinación con la karenitecina, un inhibidor de la topoisomerasa I; el resultado fue estabilización de la enfermedad en $47 \%$ (siete de 15 pacientes de la cohorte del incremento de la dosis). ${ }^{44}$ En un análisis aleatorizado de fase III que incluyó a 36 pacientes con cáncer cervicouterino avanzado, la combinación de hidralazina, AVP, cisplatino y topotecán resultó en mejoría significativa en la supervivencia libre de progresión. ${ }^{45}$ Otro trabajo in vitro demostró que el AVP aumenta la acetilación de la histona H3. Con estos cambios en las histonas, este fármaco evita la resistencia por la inhibición de mTOR por el compuesto RAD001 (everolimus) en células de carcinoma renal CaKi-1. ${ }^{46}$

\section{AVP para prevenir el cáncer de cabeza y cuello}

En un estudio retrospectivo de una cohorte de 439628 adultos mayores tratados con AVP por diferentes diagnósticos (trastorno bipolar, migraña, epilepsia) se observó menor frecuencia de carcinoma de cabeza y cuello relacionado con el tabaquismo en 26 911 individuos que utilizaron el AVP crónicamente. ${ }^{47}$

Es interesante notar que los estudios in vitro de líneas de cáncer en cultivo, in vivo con modelos animales y estudios clínicos muestran que el AVP disminuye la resistencia a la terapia convencional del cáncer. ${ }^{45,47}$

\section{Conclusiones}

En estudios de grandes poblaciones de pacientes, el AVP ha mostrado que previene el cáncer de cabeza 
y cuello; además, algunos estudios clínicos evidencian su utilidad como terapia combinada. Recientemente se ha empleado la combinación de AVP, quimioterapia y agentes que bloquean los sistemas antioxidantes (glutatión) con resultados interesantes en líneas celulares de cáncer. ${ }^{34,48}$

Los trabajos que utilizan al AVP como molécula base para diseñar nuevos compuestos se enfocan en moléculas con inhibición de HDAC relacionadas con el cáncer con poca hepatotoxicidad. Al respecto, nuestro grupo ha trabajado con el diseño y ensayo de fármacos derivados del AVP, destacando el N-(2-hidroxifenil)-2-propylpentanamide (o-OH-VPA), que ha mostrado su efecto antiproliferativo en cultivos celulares de Hela, sarcoma y MCF7.49

\section{Financiación}

Gabriela Luna Palencia es becaria del Conacyt (beca 91136) y Secretaría de Investigación y Posgrado del Instituto Politécnico Nacional, proyecto 20151399.

\section{Conflicto de intereses}

Los autores declaran que no existieron conflictos de intereses que afectaran la realización de este trabajo.

\section{Bibliografía}

1. Frazee LA, Foraker KC. Use of intravenous valproic acid for acute migraine. Ann Pharmacother. 2008:42:403-407.

2. Rosenberg $\mathrm{G}$. The mechanisms of action of valproate in neuropsychiatric disorders: can we see the forest for the trees? Cell Mol Life Sci. 2007; 64:2090-2103.

3. Hanahan D, Weinberg RA. Hallmarks of cancer: the next generation. Cell. 2011;144:646-674.

4. Dawson MA, Kouzarides T. Cancer epigenetics: from mechanism to therapy. Cell. 2012;150:12-27.

5. Tessarz P, Kouzarides T. Histone core modifications regulating nucleosome structure and dynamics. Nat Rev Mol Cell Biol. 2014:15:703-708.

6. Robey RW, Chakraborty AR, Basseville A, Luchenko V, Bahr J, Zhirong Zhan Z, et al. Histone deacetylase inhibitors: emerging mechanisms of resistance. Mol Pharm. 2011;8:2021-2031.

7. Dawson MA, Kouzarides T. Cancer epigenetics: from mechanism to therapy. Cell. 2012;150:12-27.

8. Seto E, Yoshida M. Erasers of histone acetylation: the histone deacetylase enzymes. Cold Spring Harb Perspect Biol. 2014;6:a018713.

9. Choudhary C, Kumar C, Gnad F, Nielsen ML, Rehman M, Walther TC et al. Lysine acetylation targets protein complexes and co-regulates major cellular functions. Science. 2009;325:834-840.

10. Delcuve GP, Khan DH, Davie JR. Roles of histone deacetylases in epigenetic regulation: emerging paradigms from studies with inhibitors. Clinical Epigenetics. 2012;4:5

11. De-Ruijter AJM, Van-Gennip AH, Caron HN, Kemp S, Van Kuilenburg AB. Histone deacetylases (HDACs): characterization of the classical HDAC family. Biochem. J. 2003;370:737-749.

12. Dejligbjerg M, Grauslund M, Litman T, Collins L, Qian X, Jeffers M, et al. Differential effects of class I isoform histone deacetylase depletion and enzymatic inhibition by belinostat or valproic acid in HeLa cells. Mo Cancer. 2008;7:70.

13. Witt O, Deubzer HE, Lodrini M, Milde T, Oehme I. Targeting histone deacetylases in neuroblastoma. Curr Pharm Des. 2009;15:436-447.

14. Silva MF, Aires CC, Lui PB, Ruiter JP, IJlst L, Durán M, et al. Valproic acid metabolism and its effects on mitochondrial fatty acid oxidation: a review, J Inherit Metab Dis. 2008:31:205-216.

15. Puri AS, Sharma BC, Khan EM, Saraswat VA. Fatal fulminant hepatic ailure due to sodium valproate in an adolescent. Indian Pediatr. 1994;31:207-210.

16. Ho PC, Abbott FS, Zanger UM, Chang TK. Influence of CYP2C9 genotypes on the formation of a hepatotoxic metabolite of valproic acid in human liver microsomes. Pharmacogenomics J. 2003;3:335-342.

17. Kiang TK, Ho PC, Anari MR, Tong V, Abbott FS, Chang TK. Contribution of CYP2C9, CYP2A6, and CYP2B6 to valproic acid metabolism in hepatic microsomes from individuals with the CYP2C ${ }^{*} 1 /{ }^{*} 1$ genotype. Toxicol Sci. 2006:94:261-271.

18. Sedeque AJM, Fisher MB, Korzekwa KR, González FJ, Rettie AE. Human CYP2C9 and CYP2A6 mediate formation of the hepatotoxin 4-ene-valproic acid. J Pharmacol Exp Ther. 1997;283:698-703.

19. Fantin VR, Richon VM. Mechanisms of resistance to histone deacetylase inhibitors and their therapeutic implications. Clin Cancer Res. 2007; 13:7237-7242.

20. Olsen EA, Kim YH, Kuzel TM, Pacheco TR, Foss FM, Parker S, et al. Phase Ilb multicenter trial of vorinostat in patients with persistent, progressive, or treatment refractory cutaneous T-cell lymphoma. J Clin Oncol. 2007;25:3109-3115.

21. Bassett SA, Barnett MP. The role of dietary histone deacetylases (HDACs) inhibitors in health and disease. Nutrients. 2014;6:4273-4301.

22. McGraw AL. Romidepsin for the treatment of T-cell lymphomas. Am J Health Syst Pharm. 2013;70:1115-1122.

23. Garnock-Jones KP. Panobinostat: first global approval. Drugs. 2015; 75:695-704.

24. Mercurio C, Minucci S, Pelicci PG. Histone deacetylases and epigenetic therapies of hematological malignancies. Pharmacol Res. 2010;62:18-34.

25. Ghosh SK, Perrine SP, Williams RM, Faller DV. Histone deacetylase inhibitors are potent inducers of gene expression in latent EBV and sensitize lymphoma cells to nucleoside antiviral agents. Blood. 2012; 119:1008-1017

26. Fraga MF, Ballestar E, Villar-Garea A, Boix-Chornet G, Espada J, Schotta $\mathrm{G}$, et al. Loss of acetylation at Lys16 and trimethylation at Lys20 of histone $\mathrm{H} 4$ is a common hallmark of human cancer. Nat Genet. 2005; 3:391-400.

27. Thurn KT, Thomas S, Moore A, Munster PN. Rational therapeutic combinations with histone deacetylase inhibitors for the treatment of cancer. Future Oncol. 2011;7:263-283.

28. Kirschbaum MH. Histone deacetylase inhibitors and Hodgkin's lymphoma Lancet Oncol. 2011:12:1178-1179.

29. Zapotocky M, Mejstrikova E, Smetana K, Stary J, Trka J, Starkova J. Valproic acid triggers differentiation and apoptosis in AML1/ETO-positive leukemic cells specifically. Cancer Lett. 2012;319:144-153.

30. Iacomino G, Medici MC, Russo GL. Valproic acid sensitizes K562 erythroleukemia cells to TRAIL/Apo2L-induced apoptosis. Anticancer Res. 2008;28:855-864.

31. Göttlicher M, Minucci S, Zhu P, Krämer OH, Schimpf A, Giavara S, et al. Valproic acid defines a novel class of HDAC inhibitors inducing differentiation of transformed cells. EMBO J. 2001;20:6969-6978.

32. Phiel CJ, Zhang F, Huang EY, Guenther MG, Lazar MA, Klein PS. Histone deacetylase is a direct target of valproic acid, a potent anticonvulsant, mood stabilizer, and teratogen. J Biol Chem. 2001;276:36734-36741.

33. Khan N, Jeffers M, Kumar S, Hackett C, Boldog F, Khramtsov N, et al. Determination of the class and isoform selectivity of small-molecule histone deacetylase inhibitors. Biochem J. 2008;409:581-589.

34. Bermúdez-Lugo JA, Perez-Gonzalez O, Rosales-Hernández MC, Ilizaliturri-Flores I, Trujillo-Ferrara J, Correa-Basurto J. Exploration of the valproic acid binding site on histone deacetylase 8 using docking and molecular dynamic simulations. J Mol Model. 2012;18:2301-2310.

35. Münster P, Marchion D, Bicaku E, Lacevic M, Kim J, Centeno B, et al. Clinical and biological effects of valproic acid as a histone deacetylase inhibitor on tumor and surrogate tissues: phase I/II trial of valproic acid and epirubicin/FEC. Clin Cancer Res. 2009;15:2488-2496.

36. Mohammed TA, Holen KD, Jaskula-Sztul R, Mulkerin DR, Lubner SJ, Schelman WR, et al. A pilot phase II study of valproic acid for treatment of low-grade neuroendocrine carcinoma. Oncologist. 2011;16:835-843.

37. Kuendgen A, Knipp S, Fox F, Strupp C, Hildebrandt B, Steidl C et al. Results of a phase 2 study of valproic acid alone or in combination with all-trans retinoic acid in 75 patients with myelodysplastic syndrome and relapsed or refractory acute myeloid leukemia. Ann Hematol. 2005; 84:61-66

38. Pilatrino C, Cilloni D, Messa E, Morotti A, Giugliano E, Pautasso M, et al. Increase in platelet count in older, poor-risk patients with acute myeloid leukemia or myelodysplastic syndrome treated with valproic acid and all-trans retinoic acid. Cancer. 2005;104:101-109.

39. Fenaux P, Mufti GJ, Hellstrom-Lindberg E, Santini V, Finelli C, Giagounidis $\mathrm{A}$, et al. Efficacy of azacitidine compared with that of conventional care regimens in the treatment of higher-risk myelodysplastic syndromes: a randomised, open-label, phase III study. Lancet Oncol. 2009; 10:223-232 
40. Fenaux P, Mufti GJ, Hellström-Lindberg E, Santini V, Gattermann N, Germing U, et al. Azacitidine prolongs overall survival compared with conventional care regimens in elderly patients with low bone marrow blast count acute myeloid leukemia. J Clin Oncol. 2010;28:562-569.

41. Raffoux E, Cras A, Recher C, Boëlle PY, De Labarthe A, Turlure P, et al. Phase 2 clinical trial of 5 -azacitidine, valproic acid, and all-trans retinoic acid in patients with high-risk acute myeloid leukemia or myelodysplastic syndrome. Oncotarget. 2010:1:34-42.

42. Münster P, Marchion D, Bicaku E, Schmitt M, Lee JH, DeConti R, et al. Phase I trial of histone deacetylase inhibition by valproic acid followed by the topoisomerase II inhibitor epirubicin in advanced solid tumors: a clinical and translational study. J Clin Oncol. 2007:25:1979-1985.

43. Scherpereel A, Berghmans T, Lafitte JJ, Colinet B, Richez M, Bonduelle $\mathrm{Y}$, et al. Valproate-doxorubicin: promising therapy for progressing mesothelioma. A phase II study. Eur Respir J. 2011:37:129-135.

44. Daud Al, Dawson J, DeConti RC, Bicaku E, Marchion D, Bastien S, et al Potentiation of a topoisomerase I inhibitor, karenitecin, by the histone deacetylase inhibitor valproic acid in melanoma: translational and phase I/II clinical trial. Clin Cancer Res. 2009:15:2479-2487.

45. Coronel J, Cetina L, Pacheco I, Trejo-Becerril C, González-Fierro A, De la Cruz-Hernández E, et al. A double-blind, placebo-controlled, randomi- zed phase III trial of chemotherapy plus epigenetic therapy with hydralazine valproate for advanced cervical cancer. Preliminary results. Med Oncol. 2011;28:S540-S546.

46. Juengel E, Dauselt A, Makarević J, Wiesner C, Tsaur I, Bartsch G, et al. Acetylation of histone $\mathrm{H} 3$ prevents resistance development caused by chronic mTOR inhibition in renal cell carcinoma cells. Cancer Lett. 2012;324:83-90.

47. Kang H, Gillespie TW, Goodman M, Brodie SA, Brandes M, Ribeiro M, et al. Long-term use of valproic acid in US veterans is associated with a reduced risk of smoking-related cases of head and neck cancer. Cancer. 2014;120:1394-1400.

48. Luna-Palencia GR, Martínez-Ramos F, Vásquez-Moctezuma I, Fragoso-Vázquez MJ, Mendieta-Wejebe JE, Padilla-Martínez II, et al. Three amino acid derivatives of valproic acid: design, synthesis, theoretical and experimental evaluation as anticancer agents. Anticancer Agents Med Chem. 2014:14:984-993.

49. Prestegui-Martel B, Bermúdez-Lugo JA, Chávez-Blanco A, Dueñas-González A, García-Sánchez JR, Pérez-González OA, et al. N-(2-hydroxyphenyl)-2-propylpentanamide, a valproic acid aryl derivative designed in silico with improved anti-proliferative activity in HeLa, rhabdomyosarcoma and breast cancer cells. J Enzyme Inhib Med Chem. 2016;31:140-149. 